\title{
Design of a Simplified Data Acquisition System for Classroom Use
}

\author{
Tim Cooley, Nghia Le, Terry O'Connor, Jeff Randall \\ Purdue University, School of Technology
}

While the modern desktop computer used by students today is a valuable analytical and computational tool, it is rarely studied in the classroom as a heat generation/dissipation machine. Normally this type of investigation would involve an array of protruding sensors and other components connected to a separate computer containing the usual data acquisition hardware and software components. To minimize this complexity and provide students with a simple standalone system to study, the authors designed a software and hardware package for installation into any standard desk-top computer. This package, called the Thermal Computing System (TCS), allows students to study the thermal performance of a typical computer and its individual subsystems without reliance on any external components or software.

The TCS consists of a custom designed hardware and software package that will display, control, and record in real-time the thermal behavior of a typical desktop personal computer system and its components. The system will monitor, display, and store the temperatures at eight predetermined internal locations, and has software and hardware controls for manipulation of selected components and air flows. This system allows students to explore the actual thermal behavior of a typical desktop computer and its internal components without the need for secondary data acquisition hardware, software or computers. Additional benefits include low cost (less than \$50) and essentially no learning curve.

This paper discusses the overall design and performance of this Thermal Computing System. Sample data demonstrating the behavior of the completed computer system are included.

\section{System overview}

The overall system consists of a standard tower-style IBM-clone computer circa mid 1990s, the Thermal Computing System (TCS) housed in the computer's original CD case, and a Visual Basic program written to simplify operator use of the TCS.

The base computer initially contained the usual components: an AT Form Factor Super Socket 7 mother board, 32 MB RAM, a 166 Pentium 1 processor, a 2.6 GB hard drive, a 100M Zip Drive, a 1.44 M Floppy Drive, and a 12X CD drive. I/O components included an Ethernet card, an SB16 sound card, and an S3 Virge video card. The power supply was rated at $250 \mathrm{~W}$.

From this initial setup two modifications were made; one for deliberate control of cooling air through the system, and the other for containment and functionality of the TCS within the original CD drive box. First, air flow through the unit was more-specifically controlled by sealing small unintentional entry points with tape, and then by installing an adjustable flap-door over the power supply fan outlet grill. The power supply fan serves to draw air through the system, then through 
the power supply, and finally out the top rear of the case. The added flap door allows manual control (restriction up to $98 \%$ ) of this overall flow of air through the computer beginning at the remaining lower entry points.

Second, the TCS hardware package is installed within the original 12X CD drive, after removal of all original internal components. Within this box are two 2" X 5" circuit cards in a stacked arrangement, a small fan, and the original I/O sockets modified to provide power, thermocouple entry, and parallel-port communications. A two-way toggle switch is also mounted to the face plate to control the computer's CPU fan.

Standard software for overall computer operation includes Windows 98 II and Microsoft Office. This allows standard methods to be used for data file generation, formatting, and storage, as well as the other recognizable features common to windows applications (title bar, $\mathrm{min} / \mathrm{max} / \mathrm{exit}$ icons, etc). Retaining Microsoft Office also allows the resulting data files to be analyzed on-board, further leveraging its stand-alone capability.

Custom software for operator control of the Thermal Computing System was designed using Visual Basic. This software allows the operator to Start/Stop/End the application, configure it for specific test runs, apply CPU/GPU benches to "max out" these components, and display the status of operating parameters such as thermocouple temperatures, measurement units, and the scan cycle interval.

\section{TCS Hardware}

The TCS hardware package contained within the CD box is described in segments of the mechanical design of the box hardware, the thermocouples, and the electrical circuit design. Total cost for this package was less than $\$ 50$.

\section{Mechanical Design}

The CD box contains the following components, designed from the mechanical standpoint to provide the necessary structural integrity, air flow, and I/O capability. As stated above the system started with the shell from a tray-style $12 \mathrm{X} \mathrm{CD}$ drive. A 0.094 inch thick acrylic sheet is added to provide a rigid internal platform for mounting the other components. Two 2" X 5" circuit boards are mounted on the left side in a stacked arrangement containing the electrical components (described in detail below). Spacers (5/16") are used on both sides of both cards to insure adequate clearance for cooling air and electrical circuit integrity. The original rear I/O sockets are reused to provide $12 \mathrm{VDC}$ power, a sealed entry point for the 8 thermocouples, and parallel-port communications to the motherboard. Also, a two-way toggle switch is mounted on the face plate to control the CPU heat sink fan, discussed later. Its control wire also passes through the thermocouple I/O socket to insure a complete air seal between the TCS package and the cooling air circulating through the computer.

Finally, a small $10 \mathrm{cfm}$ fan similar in size to a CPU fan is mounted on the right side to provide positive air flow to all TCS components, independent of the basic computer's cooling air. This

"Proceedings of the 2003 American Society for Engineering Education Annual Conference \& Exposition Copyright (C) 2003, American Society for Engineering Education" 
isolation is necessary in order to provide an air source for the thermocouple reference junction that is completely independent of the computer's cooling air, which enters and exits from the rear of the computer case. A sealed passageway is included below the CD box to direct air from vent holes in an unused slot cover below the $\mathrm{CD}$ box directly to the circulating fan. A partition is also placed inside the CD box to direct the air from the fan over the two circuit boards before it exits through vent holes and original openings in the front panel of the CD box. This arrangement insures a reference junction temperature that is consistent with ambient air conditions surrounding the entire computer. See figure 1 below.

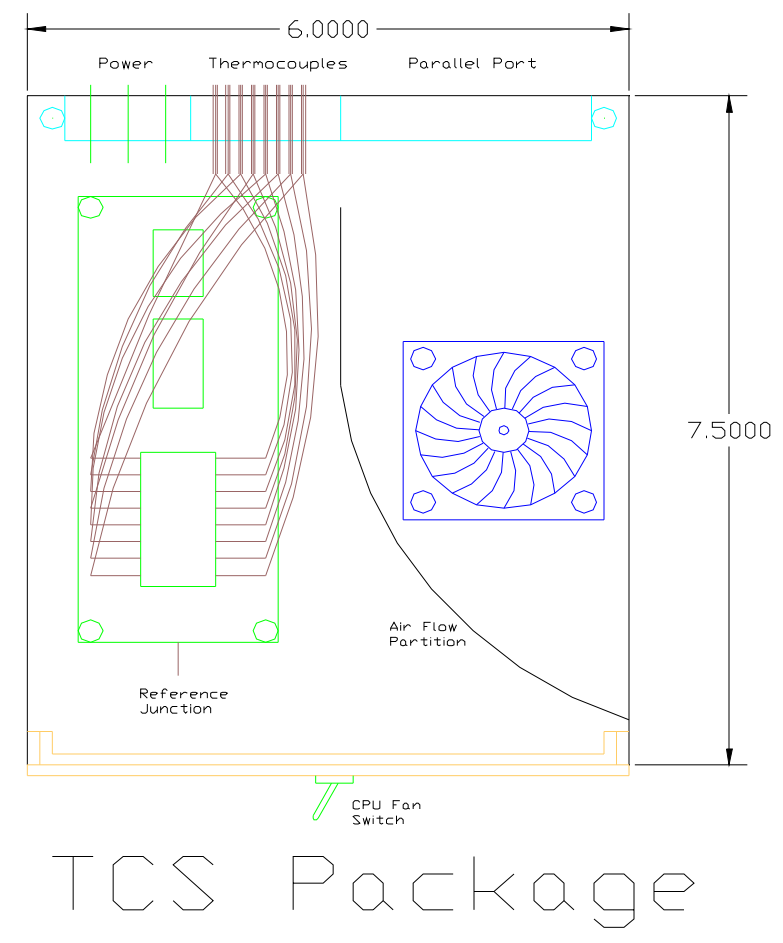

Figure 1

\section{Thermocouples}

The 8 thermocouples are Type $\mathrm{K}$ with soldered junction tips, approximately 30 inches long, and sealed at the rear entry point to the $\mathrm{CD}$ box. Sensing points within the computer include the $\mathrm{CPU} /$ heat sink interface, the heat sink/CPU fan interface, the CPU fan inlet, the graphics processing unit (GPU) top surface, the primary computer case air inlet point (the lower-most card slot opening), the hard drive surface (directly above the main drive bearing), the power supply air inlet grill, and the power supply fan outlet grill. Another thermocouple is used as the reference junction on the lower circuit board within the CD box. Techspray ${ }^{\circledR}$ heat sink compound is applied at the four thermocouple/surface interfaces to insure efficient thermal conductivity to the thermocouple junction. The four air-sensing thermocouples (computer case inlet, CPU fan inlet, power supply inlet, power supply outlet) are each placed $1 / 4$ inch into their respective air streams to minimize unintended movement and contact. 


\section{Electrical Circuit Design}

\section{General Description}

The circuit design consists of several different sections. The fundamental part of the circuit is a thermocouple signal conditioning IC. The output of the thermocouple signal conditioner IC is then fed into an Analog to Digital converter. The digital output is then inputted into the PC. A multiplexer is used to switch one of the 8 thermocouple inputs to a common output and then into the thermocouple conditioning IC. A 3-bit binary counter is used to supply a sequential count sequence of a binary 1 through 8 , which is connected to the decoder on the multiplexer. This provides a way to scan all of the thermocouple inputs. The PC is used to control the advancing of the counter. See Figure 2.

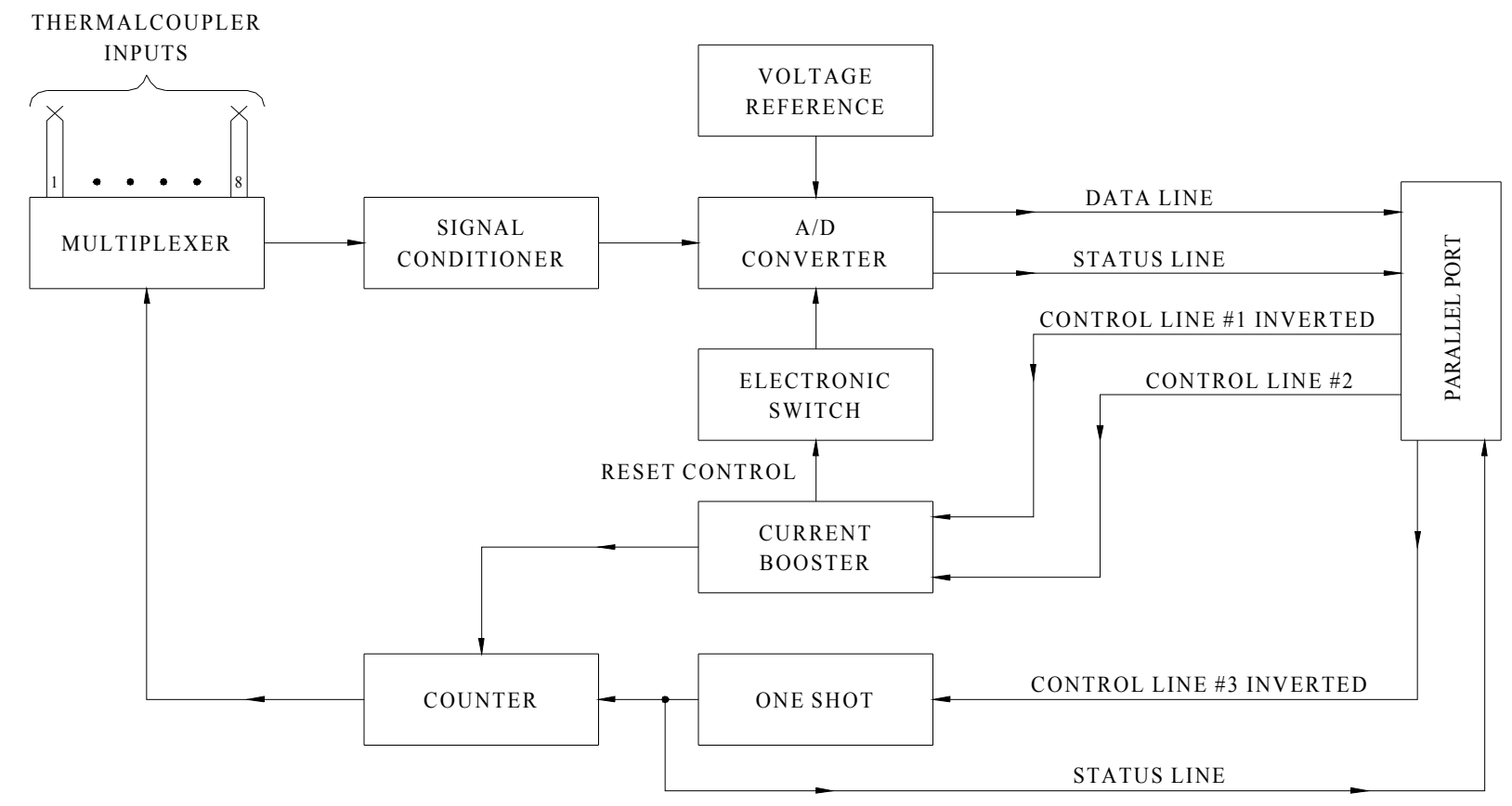

Figure 2

\section{Component Design}

An AD595, made by Analog Devices, is used to provide the thermocouple signal conditioning portion of the circuit. The AD595 is a Monolithic Thermocouple Amplifier with Cold Junction Compensation. It generates an output of $10 \mathrm{mV} / 1^{\circ} \mathrm{C}$. Used in conjunction with the AD595, is another IC manufactured by Analog Devices, the ADG507. It is a CMOS monolithic analog multiplexer with dual 8 channel inputs. The ADG507 switches one of the eight differential inputs to a common differential output. The input that is selected depends on the state of the three decoder lines. The enable input pin must be high for the device to operate. The output of the ADG507 is fed directly into the AD595 -IN input. See Figure 3 below.

The analog voltage out of the AD595 had to be converted to an 8-bit digital value to prepare it for input into the PC via the parallel port. An ADC0804 made by National Semiconductor is used for conversion. The ADC0804 is a CMOS 8-bit successive approximation A/D converter. It is 
set up to operate in free-running mode to do continuous operations. The clock is set to oscillate at $640 \mathrm{kHz}$. This provides approximately 8000 conversions per second. The analog to digital converter needs to be scaled so that for every $10 \mathrm{mV}$ step on the analog input it changes its output by a binary count of one. For example if there is a $10 \mathrm{mV}$ input into the A-D, the conversion is 00000001 , for $20 \mathrm{mV}$ it is 00000010 , for $30 \mathrm{mV}$ it is 00000011 , and so on. The temperature in degrees Celsius now corresponds to the binary value of the output of the ADC0804. The A-D converter needs to have a voltage span of $0 \mathrm{~V}$ to $2.55 \mathrm{~V}$. This is calculated by multiplying 255 possibilities in an 8-bit value by $10 \mathrm{mV}$. To accommodate the 2.55 volt range there needs to be a voltage reference of $1.275 \mathrm{~V}$. This voltage is then applied to the Vref/2 pin of the ADC 0804 . The TLV431A is used to provide a stable voltage reference. It is a Low Voltage Precision Adjustable Shunt Regulator. The output of the A-D converter is then connected to the data lines of the parallel port. An initialization pulse should be applied after power on to ensure proper operation in free-running mode. The WR and INTR pins need to be forced to a logic low. A transistor switch and a AND gate are used to accomplish this. One input on a 7408 AND gate is tied to $+5 \mathrm{~V}$ while the other is connected to a CTRL line on the parallel port. This allows control to be done from within the program. The output of the AND gate is tied to the base of the transistor. The collector of the transistor is connected to the WR and INTR pins and the emitter is tied to ground. The AND gate output is momentarily set high upon power up using the program causing the transistor to turn on and therefore grounding the WR and INTR pins. The momentary low state initializes the counter. The high impedance between the collector and emitter provides a "floating" state during the rest of the operation so it doesn't affect the state of the WR and INTR pins.

The next block of the design consists of the circuit involved in advancing the binary counter. This is important because it controls which analog input is connected to the A/D input. A 7493, which is a binary counter, is used to control the decoder on the ADG507 multiplexer. The outputs QA, $\mathrm{QB}$, and QC are connected to the A1, A2, and A3 pins of the multiplexer respectively. QD is not used because three bits are sufficient to provide a maximum count of 8 . There are two reset pins on the 7493, one reset input is tied to $+5 \mathrm{~V}$ and the other to the output of an AND gate. One input of the AND gate goes to one of the control lines of the parallel port to be able to control when the counter is reset. A logic 1 is provided to the other input of the AND gate by applying $+5 \mathrm{~V}$ to it. In this manner the AND gate is serving as a buffer. When a logic 1 is applied by the parallel port the counter resets. A one-shot is used to advance the counter. The chip that is used is a 74221. The Q output of the one-shot is connected to the Clock A input of the counter. The one-shot is setup to have a $\mathrm{Q}$ output pulse width of approximately $76 \mathrm{~ms}$ to give adequate time to allow for an A/D conversion of the analog channel connected to the AD595. The counter advances by a count of one when a pulse from the one-shot is applied to the counters clock B input. One of the control lines of the parallel port is connected to the B input of the one-shot to control when the counter advances.

The last component involved in the circuit design is the parallel port. The PC BIOS needs to be set to EPP mode for proper operation of the parallel port for this project. Parallel ports use three $\mathrm{I} / \mathrm{O}$ addresses. There is the data register, status register, and control register with addresses of $378 \mathrm{~h}, 379 \mathrm{~h}$, and $380 \mathrm{~h}$ respectively. The port has 8 data lines, 5 status lines, and 4 control lines.

"Proceedings of the 2003 American Society for Engineering Education Annual Conference \& Exposition Copyright (C) 2003, American Society for Engineering Education" 
This project uses all 8 data lines which are bi-directional, 2 status lines that are read only lines, and 3 control lines which are write only. Pins $18-25$ are grounded. The 8 data lines are connected to the A/D converter's digital output. Status line 3 is connected to the ADC0804's interrupt and write pins to detect when the conversion processes are to complete. Status line 4 is connected to the 7493 to detect when the trigger to advance the counter has finished its cycle. Control line 3 is connected to the 74221's input pulse to trigger the one-shot. This line is an inverted pin so that had to be adjusted in the program to get the desired output. Control lines 1 and 2 are both connected to the 7408 AND gate. One is used to reset the counter when needed and the other is used to reset the $\mathrm{A} / \mathrm{D}$ converter upon power up. Control line 1 is also inverted.

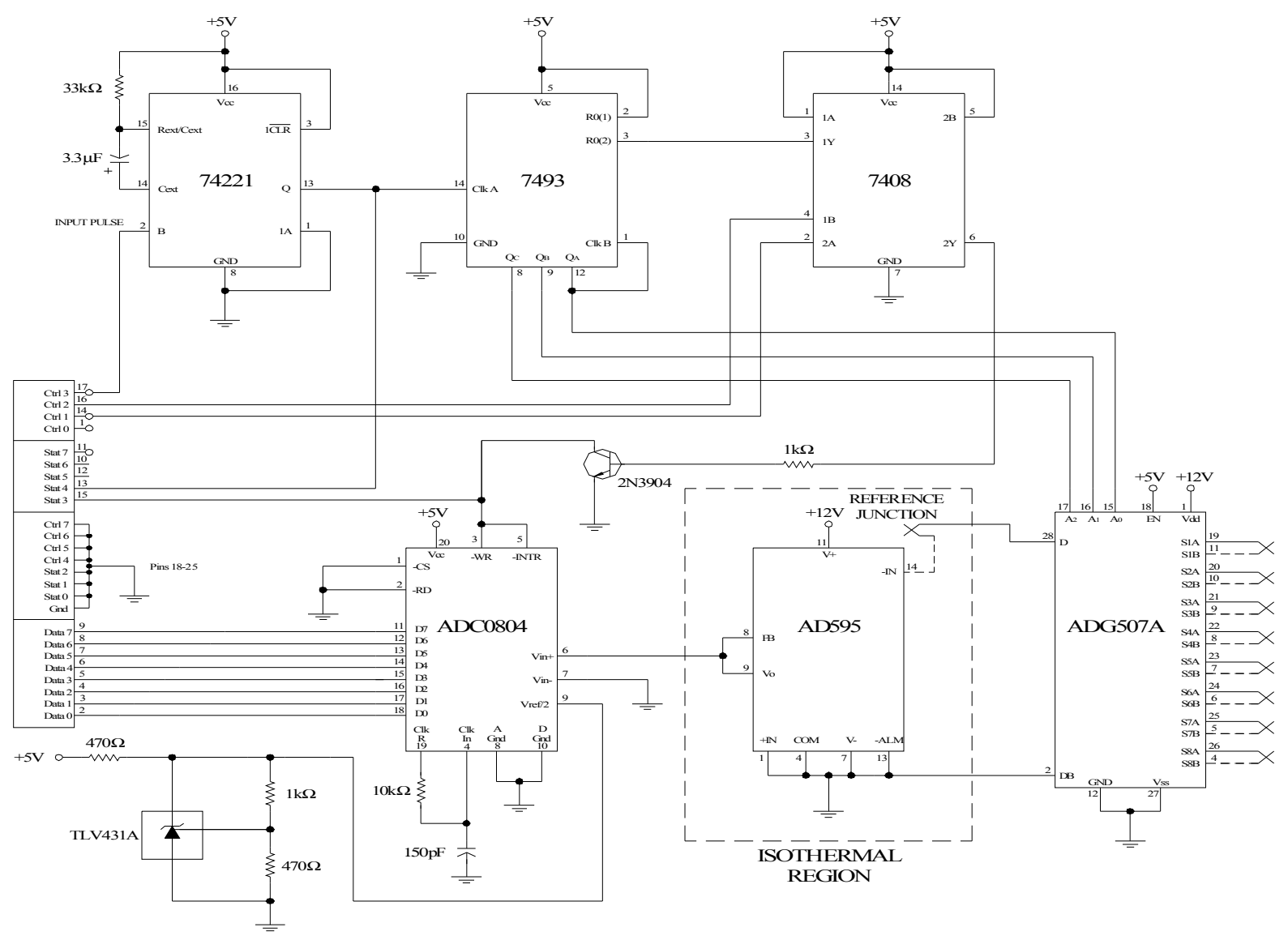

Figure 3

\section{Operational Description}

All control and data computation is accomplished by using Microsoft Visual Basic. The following paragraphs will describe the steps involved to acquire the data and how each is done.

The first thing that happens when the program is executed is the initialization of the $A / D$ converter and the resetting of the binary counter. A logic low is sent to control line 1 of the parallel port. This signal then goes to the 7408 AND gate. Since the control line is inverted, a logic high is applied to the AND gate. The other input of the gate is tied to a logic high, therefore

"Proceedings of the 2003 American Society for Engineering Education Annual Conference \& Exposition Copyright (C) 2003, American Society for Engineering Education" 
the output will go high causing the transistor to turn on. The interrupt and write pins of the A-D converter will be at a logic low when the transistor turns on and this will cause the A-D converter to initialize for proper operation. A logic high is sent via control line 2 of the parallel port to reset the counter. This signal is buffered by the 7408 AND gate. The other input is tied high so this causes a logic high on the output. One of the reset lines of the 7493 binary counter is connected to the output of the AND gate and when this line goes high the counter will reset its count registers.

The next step is the reading of the data at the $\mathrm{A} / \mathrm{D}$ converter data lines. The first thing that is done in this step is to read status line 3 to see if the interrupt pin on the A/D converter is high. The interrupt pin signals the end of a conversion by going high. Once the status line ensures the pin is high the flow of the program continues. The data is now ready to be read into the PC via the data lines.

The final step of the program is to evaluate the counter. If the counter is greater than 8 then it is reset to begin counting from 0 again. If the counter is less than 8 then the counter is advanced by one. The outputs of the counter control which line of the multiplexer is to be converted. The status of the counter register is determined by using a variable in the program to track where it is in its sequence. A logic low is applied to control line 3 of the parallel port to advance the counter. This line is inverted so a logic high will, in essence, be applied to the IC it's connected to. Since this line is connected to the input pulse for the one-shot a logic high will cause it to trigger a lowto-high transition on the output which will in turn advance the counter. The counter is reset when its count is 8 or higher. A logic high is applied by control line 2 of the parallel port to reset the counter. This causes a high at one input of the AND gate. When it goes high the output goes high since the other input is always high. The counter will now be reset.

\section{TCS User Software}

The user program is written in Visual Basic 6.0 with a matchstick icon used to activate the TCS system. The program opens and displays the Thermocouple Reader window from which the running displays and other windows are activated. Figure 4 shows the user displays discussed below. The internal data storage operation is then discussed.

\section{Main Window Components \& Functions}

Data display boxes

8 windows are shown, each displaying the last scanned temperature, in its configured units. Labels are included below each temperature window.

\section{Start/Stop/Exit buttons}

Start creates and labels a new data storage file, initializes it, and activates the display, scan, and storage routines. The file name is subsequently displayed in the main window's title bar.

\footnotetext{
"Proceedings of the 2003 American Society for Engineering Education Annual Conference \& Exposition Copyright (C) 2003, American Society for Engineering Education"
} 
Stop deactivates the application routine, labels the last line of the data file then closes it, and holds the last displayed temperature values. The main program window remains and can be activated again with the Start button.

Exit closes the TCS application and returns to Windows 98.

Config button

The Config button invokes a separate window to allow the user to separately label the individual temperature displays and titles, set temperature values in units of either Fahrenheit or Celsius, and determine the interval between temperature updates in whole numbers of from 5 to 65 seconds. Each scan cycle consists of the sequential measurement, display and storage of all 8 thermocouples as well as a calculated delay interval that, together equal the prescribed cycle time.

Bench CPU \& Bench Video buttons

Bench CPU is designed to run the central processing unit (CPU) at maximum by performing a repetitive calculation and updating its instantaneous process rate display (BPS) every second. This benching operation is in addition to any other computational demand (such as the TCS package and display) required.

Bench video is designed to run the graphics processing unit (GPU) at maximum by drawing colored lines of random length and direction within a separate display box. Both the CPU and GPU bench display boxes may be actively dragged to any onscreen location, and the GPU bench box may be sized as needed at any time.

About button

This is merely a credit window designating subsystem principals.

$\underline{\text { Status box }}$

This lower segmented box shows which thermocouple is actively being scanned and its temperature, the delay interval between scan cycles, and the data storage file name. 

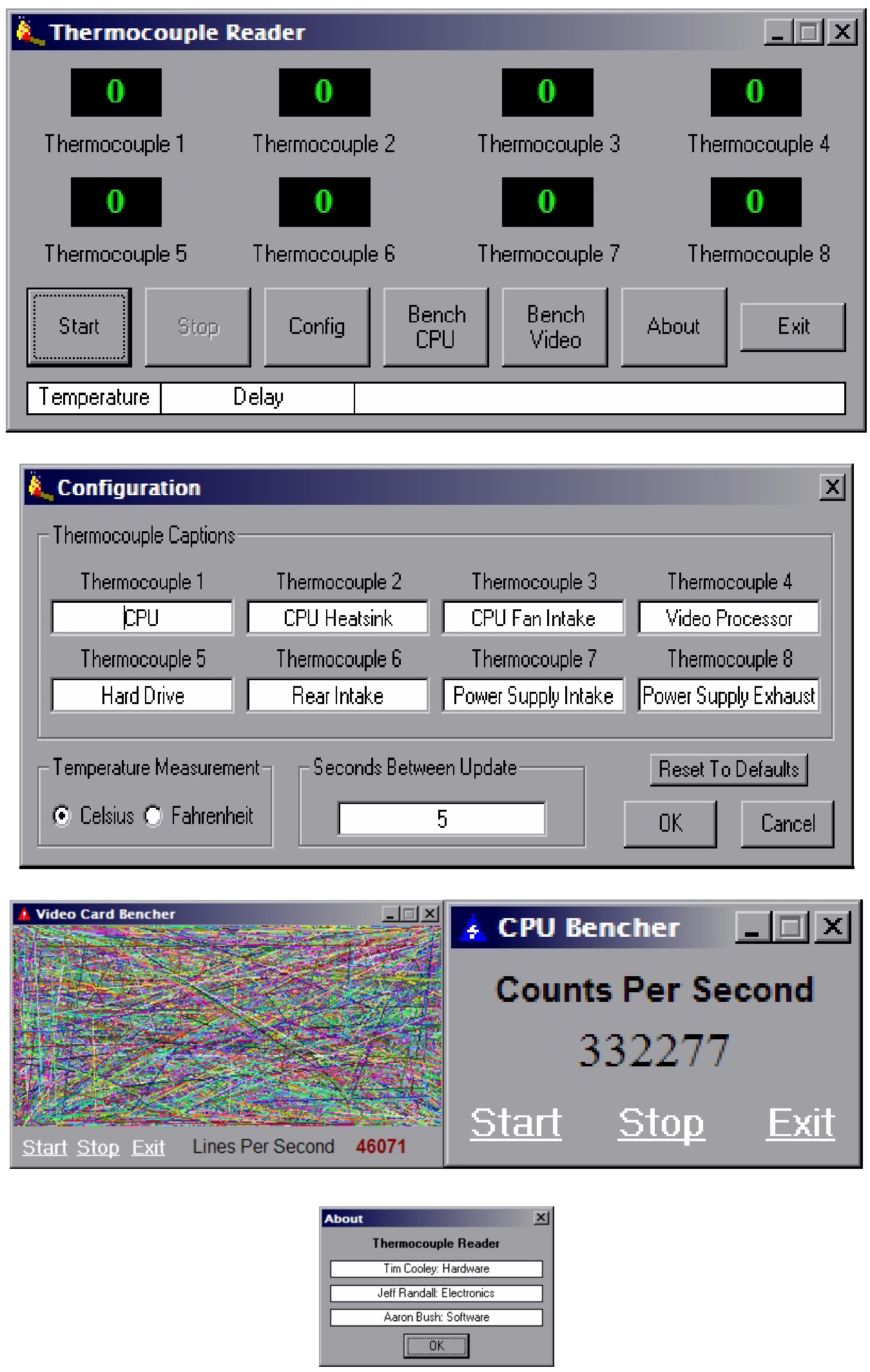

Figure 4

"Proceedings of the 2003 American Society for Engineering Education Annual Conference \& Exposition Copyright (C) 2003, American Society for Engineering Education" 


\section{Data Storage}

The data recording \& storage operation begins when the Start button is activated. A file with the informative name of [year-month-day (hour_minute_second).dat] is created and stored in the Logs directory. Within this data file the program creates a first line stating the exact time the file starts collecting data, then a new line for each scan cycle consisting of a time stamp ( $\mathrm{h}$ m_ $\mathrm{s}$ ) and each measured thermocouple temperature, with a single space separating each data value. When the Stop button is activated the program adds a last line to the file stating the exact time the button was activated (again in units of $h \_m \_s$ ), after the last remaining scan cycle is completed. It then closes the data file and returns control to the main TCS window.

A flowchart outlining the overall operation of the program is shown below.

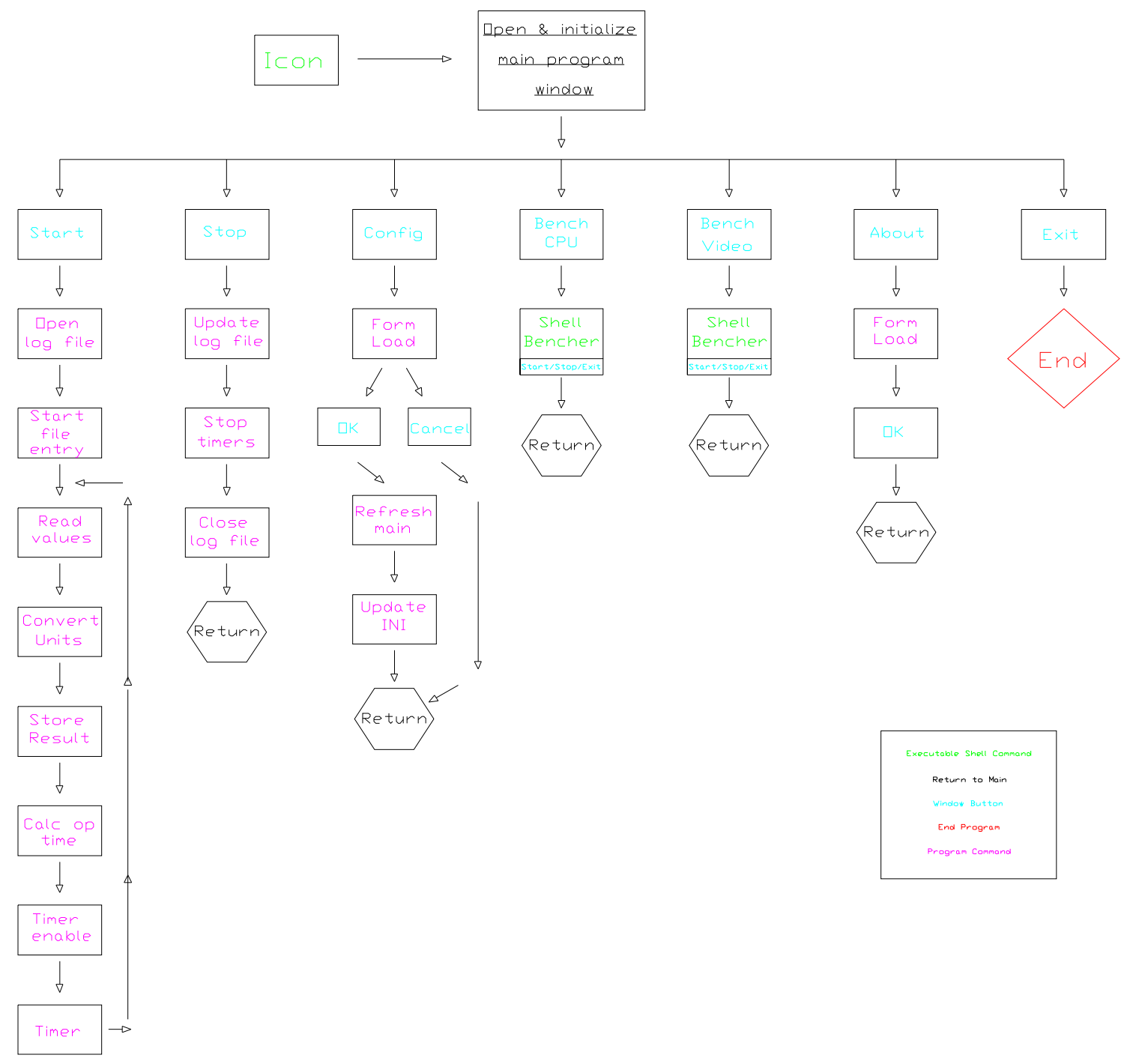

Figure 5

"Proceedings of the 2003 American Society for Engineering Education Annual Conference \& Exposition Copyright (C) 2003, American Society for Engineering Education" 


\section{Computer System Performance}

Results from a typical test run are shown below (Figure 6). Microsoft Excel was used to chart the stored values.

In this run the CPU fan was turned off and the CPU and video benches activated approximately one minute into the run for a period of 9 minutes 30 seconds. The computer's main cooling air flow (controlled with the power supply fan flap door) was not restricted.

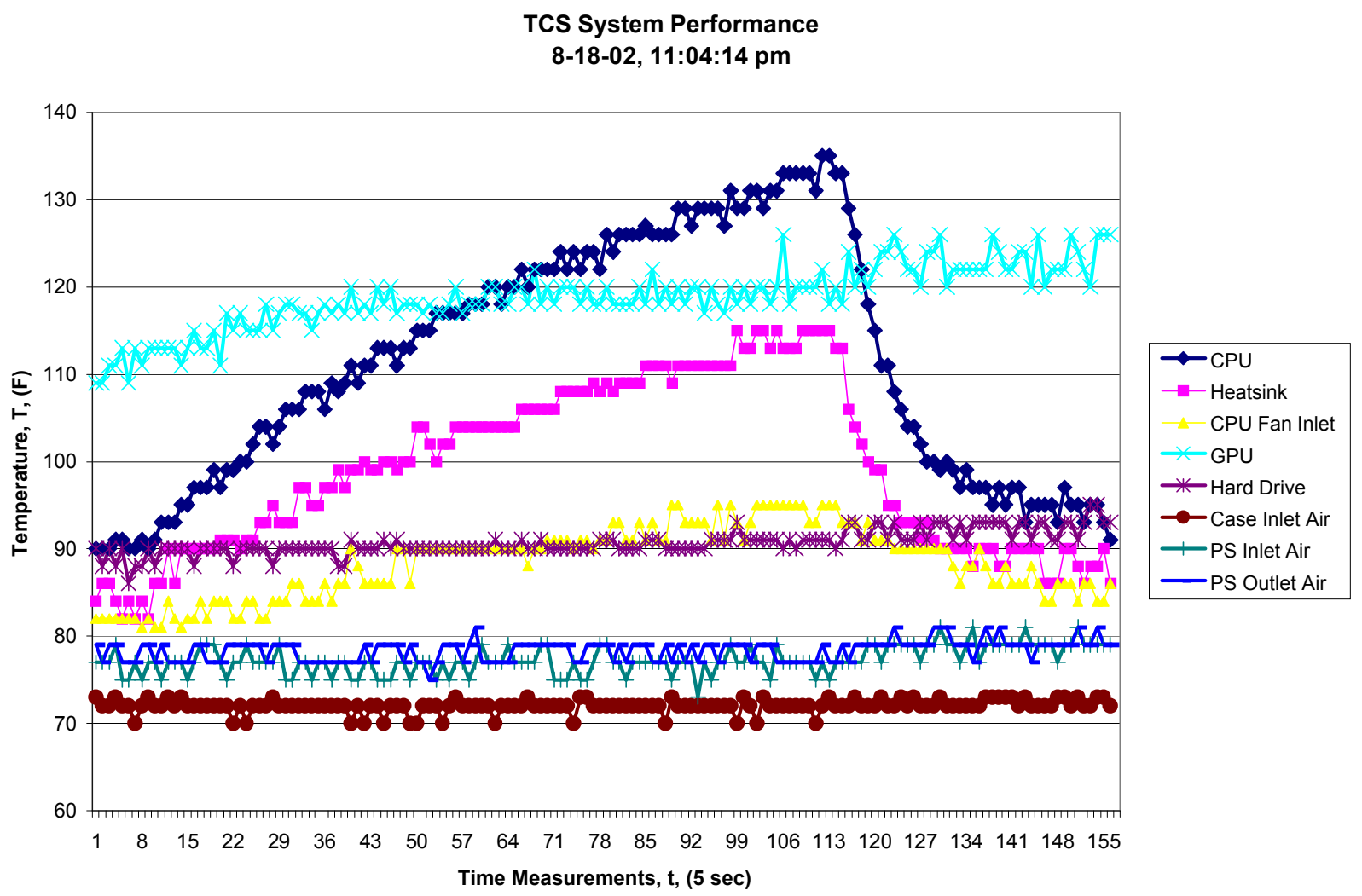

Figure 6

Several trends are apparent. First and most obviously, with the CPU fan deactivated there is an immediate rise in temperature for all three thermocouples in the immediate vicinity of the CPU. These thermocouples show a temperature rise towards a higher (but unattained in the interest of time and equipment longevity) equilibrium temperature. They also show a rapid response in returning to their baseline temperatures (also unattained in this test run). Second, the power supply inlet and outlet temperatures are virtually identical, indicating there is virtually no heat generated specifically in this device. Third, once the CPU fan is reactivated, the heat generated during the time it was off is liberated from the immediate vicinity and begins moving towards the other components, especially upwards towards the hard drive and the power supply. The GPU 
also shows an increase in not only its temperature, but also its variability, as the transient heat load begins equilibrating.

\section{Potential Applications}

As the graph in Figure 5 demonstrates, there are many heat transfer phenomena at work inside a computer system that may be appropriate for students. Testing has shown the TCS/computer system to be capable of monitoring 8 important aspects involved in the operation of a typical computer; the two primary processing units (CPU \& GPU), the primary information storage system (hard drive), the system for dissipation (heat sink \& fan) of the largest source of heat, the main power supply (inlet \& outlet), and the ambient temperature used to cool the entire system. In addition, there are 4 mechanisms designed to control component and/or system thermal performance. These controls; two that drive circuit loads, and two that control convective cooling rates, provide the means to control and test several important internal functions computers are designed to provide.

Using this TCS/computer package, students can begin to examine and analyze computers as heat generation/dissipation machines, not just as the computational or information gathering machines they are always assumed to be. And although it was designed for use by students in the study of practical applications of heat generation and transfer, it could also be used for a more rigorous, theoretical engineering-type examination. The issue that must be resolved, however, is which approach is most appropriate for the engineering technology students intended. 